\title{
Impact of Chronic Tramadol Intake on Reproductive Hormones and Related Histopathological Changes in Adult Albino Rats
}

\author{
Mona EL-Qotb Moussa, Nahla Hassan Tolba, Soha Khaled Ashry, Asmaa Mohamed Ahmed Ali, ${ }^{1}$ Suzi Sobhi Atallah ${ }^{2}$ \\ ${ }^{1}$ Forensic Medicine and Clinical Toxicology Department. \\ ${ }^{2}$ Histology Department. Faculty of Medicine, Ain Shams University, Cairo, Egypt.
}

\begin{abstract}
Background: Tramadol is a synthetic opioid analgesic, widely used in the treatment of osteoarthritis, back pain, sports injuries, and other musculo-skeletal conditions, rheumatological conditions, and headaches. Tramadol is a common prescription pain reliever as it is considered to possess a much better safety profile than other opioid analgesics.

Aim of the Work: To examine the influence of chronic use of tramadol on reproductive hormones, and to outline the histopathological changes in ovaries, testes and pituitary glands of adult albino rats.

Materials and Methods: A number of 90 male and female adult albino rats were used for this study. They were divided into three groups each comprising 30 rats (15 males and 15 females). Group one was the control group which received normal saline, group two was treated with low dose of tramadol $(40 \mathrm{mg} / \mathrm{kg})$ daily, while group three was treated with high dose of tramadol (80 $\mathrm{mg} / \mathrm{kg}$ ) daily for one month.

Results: The present study revealed that there was a statistical significant decrease in serum LH, FSH, Estradiol among tramadol treated male and female groups when compared with control group. In addition, there was a significant decrease in serum testosterone level among tramadol treated male groups when compared to the control group. Furthermore, serum progesterone level showed a statistically significant decrease among the high dose tramadol treated female group when compared to both the low dose tramadol treated group and the control group but there was a statistically significant increase when comparing the low dose to the control group and the high dose group. While monitoring the effect of tramadol on serum prolactin level there was significant increase among tramadol treated male groups, while there was non significant change among tramadol treated female groups. By histopathological examination of ovaries and testes under light microscopy and pituitary glands by electron microscopy there were changes related to the hormonal changes.

Conclusion: Chronic use of tramadol in rats even with its therapeutic doses has a passive effect on gonadal function affecting both males and females.
\end{abstract}

Key words Tramadol - Hormones - Histopathological Changes

\section{Introduction}

$\mathrm{O}$ piates are natural products derived from opium which is present in Papaver Somniferum fruit. They include morphine, codeine, thebaine and many semi-synthetic congeners derived from them. Tramadol is a synthetic analogue of codeine. The term opioid applies to any substance, whether natural or synthetic, that produces morphine like effects through an action on opioid receptors (Pasternak, 2001).

Opioids including tramadol are used as analgesics for the treatment of severe pain (acute or chronic) as cancer and non-cancer pain. According to the Centers for Disease Control and Prevention (CDC), there has been a 300\% increase in the sale of opioid analgesics from 1999 to 2011 (Egan and Newberry, 2018).

In 2006, statistics in the United States showed that approximately $90 \%$ of patients with chronic pain receive opioids (Trescot et al., 2006), and 90\% of patients presenting to an interventional pain management center are already on opioids (Manchikanti, 2004). 
It is estimated that the prevalence of a substance abuse disorder is $8.1 \%$ in the general population and even higher in the population of patients with chronic pain. These facts explain the dramatic rise in prescription drug abuse and in complications secondary to controlled substances (Trescot, 2006).

Egypt has one of the highest death rates due to road accidents and tramadol abuse is associated with $18.7 \%$ of these accidents (Bassiony et al., 2015).

The effects of long-term opioid use/abuse on the endocrine system have not been well studied because most studies on the effects of opioids on the endocrine system focus on acute effects. The effects of opioid compounds on hormonal function is now fairly well understood (Daniell, 2006). However, long- term effects at cellular level are not clearly understood (Daniell, 2002).

In addition, signs of opioid endocrinopathy often go unreported and therefore are not monitored clinically (Gudin et al., 2015).

Furthermore, most studies focus on the acute tramadol toxicity with poor information concerning its chronic use/abuse especially regarding reproductive system (El-Ghawet, 2015).

Thus, the current study was designed to investigate the effects of chronic tramadol use on reproductive system in males and females.

\section{Aim of the Work}

To investigate the effects of chronic use of tramadol on reproductive hormones and to outline the histopathological changes in ovaries, testes and pituitary glands of adult albino rats.

\section{Materials and methods}

This experimental study was carried out in the Medical Research Center, Ain Shams University on groups of adult albino rats where the effects of tramadol on reproductive system were evaluated.

\section{A- Chemical compound (tramadol)}

Commercially available Tee-Doll tablets. Each tablet contains $225 \mathrm{mg}$ tramadol hydrochloride (TeeDoll ${ }^{\circledR}$, manufactured by Minapharm, Heliopolis, Cairo, Egypt under license of German Grunenthal Company).

\section{B- Experimental design:}

\section{(A) The animals}

Ninety adult albino rats of both sexes weighing approximately $150 \mathrm{~g}$ each, were chosen for this study. They were housed in cages under controlled conditions of temperature and humidity.

At the start, all rats were left in their housing for one week, for adaptation to the environmental condition. All animals received a balanced diet containing essential elements and vitamins. Clean containers were supplied with tap water throughout the experiment.

\section{(B) Groups:}

The rats were divided into the following groups where each group comprised 30 rats.

\section{I) Group one: (Control group)}

This group was divided into 2 subgroups and received normal saline orally.

- Sub-group: control males $(\mathrm{n}=15)$

- Sub-group: control females $(\mathrm{n}=15)$

\section{II) Group two: (low dose group)}

This group was divided into 2 subgroups and both received oral doses of tramadol suspended in saline solution equal to $40 \mathrm{mg} / \mathrm{kg}$ body weight for thirty consecutive days (Senay et al., 2003).

- Sub-group: males $(\mathrm{n}=15)$

- Sub-group: females $(\mathrm{n}=15)$

III) Group three: (high dose group):

This group was divided into 2 subgroups and both received oral doses of tramadol suspended in saline solution equal to $80 \mathrm{mg} / \mathrm{kg}$ body weight for thirty consecutive days (Senay et al., 2003).

- Sub-group: males $(\mathrm{n}=15)$.

- Sub-group: females $(\mathrm{n}=15)$.

At the end of treatment, the animals were sacrificed and blood and tissue samples were collected.

\section{A) Serum samples:}

Blood samples were collected in plain tubes, left to stand in $37^{\circ} \mathrm{C}$ for 15 minutes then centrifuged at 3000 r.p.m for 10 minutes and stored at $-20^{\circ} \mathrm{C}$ until used.

\section{B) Tissue Specimen:}

The ovaries, testes and pituitary glands were dissected out and kept in 10\% formalin for histopathological studies.

\section{(C) Druq:}

\section{Preparation and dose:}

Just before its use, it was reconstituted with normal saline $(0.9 \%)$. Tramadol tablet $(225 \mathrm{mg})$ was reconstituted in $50 \mathrm{ml}$ saline $(0.9 \%)$.

Two groups of adult albino rats (30 rats each) were given orally two different doses of the reconstituted drug. Group II was given $1.3 \mathrm{ml}$ of the reconstituted drug which is equal to $40 \mathrm{mg} / \mathrm{kg}$ and group III was given 2.6 $\mathrm{ml}$ of the reconstituted drug which is equal to $80 \mathrm{mg} / \mathrm{kg}$ (Senay et al., 2003). The dose was modified every week according to the related changes in body weight.

\section{Time and route of administration:}

Tramadol was administered once daily for each animal in group two and group three for 30 consecutive days. It was administered orally by a curved needle like oral tube that was introduced directly into the stomach (a gavage process).

\section{By the end of the study the following was done:}


I) Physical changes in the weight before and after the study were monitored.

II) Laboratory investigations using enzyme linked immunosorbent assay:

The following biochemical parameters had been measured:

\section{A) For female groups:}

The serum levels of luteinizing hormone ( $\mathrm{LH})$, follicle stimulating hormone (FSH), estradiol (E2), progesterone and prolactin were measured.

\section{B) For male groups:}

The serum levels of luteinizing hormone ( $\mathrm{LH})$, follicle stimulating hormone (FSH), testosterone, estradiol (E2) and prolactin were measured.

III) Histological examination for ovaries, testes and pituitary glands:

A. Light microscopic examination for ovaries and testes:

Following rats sacrificing, the organs were rapidly dissected and fixed in neutral buffered formaldehyde solution (10\%) for 48 hours before being embedded in paraffin wax. Sections of 5 microns thickness were mounted on glass slides and stained with hematoxylin and eosin (Hx \& Ex) according to method adopted by Drury and Wallington (1980) and examined by light microscope for histopathological investigation.

\section{B. Electron microscopic examination for pituitary glands:}

Specimen preparation and stains used:

This was carried out in the Electron Microscope Unit, Faculty of Science, Ain Shams University. Pituitary gland tissues of all groups were fixed in formol glutaraldehyde to be processed for electron microscopic study.

After being embedded, semi-thin sections $(1 \mu \mathrm{m}$ thick) were cut using an ultra microtome. They were stained with toluidine blue, and examined by the light microscope. Then, ultra-thin sections (60-90 nanometers) were cut using the ultra microtome, mounted on copper girds and stained with uranyl acetate and lead citrate (Luft, 1961).

\section{Statistical analysis}

Data were collected, checked, revised and analyzed by SPSS statistical package version 19. Excel computer program was used to tabulate the results, and represent it graphically. One Way ANOVA was used to declare the significant difference between groups at $\mathrm{p}<0.05$ for the quantitative variables which are normally distributed, and TUKEY'S comparisons test at $\mathrm{p}<0.05$ was used to declare the significant difference between each two groups.

\section{Results}

I. Laboratory parameters

1. Female groups

Serum prolactin in studied female groups

Table (1) shows the values of serum prolactin. Statistical analysis by using one way ANOVA test revealed that there was non-significant difference between female groups as regards serum prolactin.

\section{Serum LH in studied female groups}

Table (2) shows the values of serum LH. Statistical analysis by using one way ANOVA test revealed that there was a significant difference between the female groups as regards serum LH.

On applying TUKEY'S comparison test, serum LH showed a statistical significant decrease in group II and group III when compared to group I, while there was non significant difference in LH decrease between group II and group III.

\section{Serum FSH in studied female groups}

Table (3) shows the values of serum FSH. Statistical analysis by using one way ANOVA test revealed that there was a significant difference between female groups as regards serum FSH.

On applying TUKEY'S comparison test, serum FSH showed a statistical significant decrease in group II and group III when compared to group I. In addition, group III showed statistical significant decrease in serum FSH when compared to group II as regards serum FSH.

\section{Serum Estradiol (E2) in studied female groups}

Table (4) shows the values of serum Estradiol. Statistical analysis by using one way ANOVA test revealed that there was a significant difference between female groups as regards serum Estradiol.

On applying TUKEY'S comparison test, serum Estradiol showed a statistical significant decrease in group II and group III when compared to group I. On the other hand, group III showed statistical significant decrease in serum Estradiol when compared to group II.

\section{Serum progesterone in studied female groups}

Table (5) shows the values of serum progesterone. Statistical analysis by using one way ANOVA test revealed that there was a significant difference between the groups under comparison.

On applying TUKEY'S comparison test, serum progesterone showed a statistical significant increase in group II when compared to group I and group III. On the other hand, group III showed a statistical significant decrease in serum progesterone when compared to group I and group II. 
Weight estimation before and after the experiment in female groups

Table (6) shows the weight of studied female groups before and after the experiment. Statistical analysis by using one way ANOVA test revealed that there was a significant difference between female groups as regards weight after the experiment, while there was a nonsignificant difference between female groups as regards weight before the experiment. On applying TUKEY'S comparison test, there was non-significant difference in weight between group I and group II after the experiment, while there was a statistical significant decrease in weight in group III when compared with group I and group II.

\section{Male groups}

\section{Serum prolactin in studied male groups}

Table (7) shows the values of serum prolactin. Statistical analysis by using one way ANOVA test revealed that there was a significant difference between male groups as regards serum prolactin.

On applying TUKEY'S comparison test, serum prolactin showed a significant increase in group II and group III when compared to group I, while there was a non-significant difference in prolactin level between group II and group III.

\section{Serum LH in studied male groups}

Table (8) shows the values of serum LH. Statistical analysis by using one way ANOVA test revealed that there was a significant difference between male groups as regards serum $\mathrm{LH}$.

On applying TUKEY'S comparison test, serum LH showed a statistical significant decrease in group II and group III when compared to group I, while there was a non-significant difference in LH level between group II and group III.

\section{Serum FSH in studied male groups}

Table (9) shows the values of serum FSH. Statistical analysis by using one way ANOVA test revealed that there was a significant difference between male groups under comparison.

On applying TUKEY'S comparison test, serum FSH showed a statistical significant decrease in group II and group III when compared to group I. In addition, group III showed a statistical significant decrease in serum FSH when compared to group II.

\section{Serum Estradiol (E2) in studied male groups}

Table (10) shows the values of serum Estradiol. Statistical analysis by using one way ANOVA test revealed that there was a significant difference between male groups as regards serum Estadiol.

On applying TUKEY'S comparison test, serum Estradiol showed a statistical significant decrease in group III when compared to group I and group II, while there was a non-significant difference between group I and group II as regards serum Estrtadiol.

Table (11) shows the values of serum testosterone. Statistical analysis by using one way ANOVA test revealed that there was a significant difference between male groups under comparison.

On applying TUKEY'S Comparison Test, serum testosterone showed a statistical significant decrease in group III when compared to group I and group II, while there was a non-significant difference between group I and group II.

\section{Weight estimation before and after the experiment in} male groups

Table (12) shows weight of studied male groups before and after the experiment. Statistical analysis by using one way ANOVA test revealed that there was a significant difference between male groups as regards weight after the experiment, while there was nonsignificant difference between male groups as regards weight before the experiment.

On applying TUKEY'S comparison test, weight after the experiment showed a statistical significant decrease in group II and group III when compared to group I. On the other hand, group III showed a statistical significant decrease in weight when compared to group II.

\section{Histopathological Results}

\section{Histopathological results of the ovary:}

Light microscopic examination for ovaries of low dose female group revealed abundant fibroblasts with elongated nuclei of fibroblast proliferation, atretic follicles, congestion between the follicles and aggregation of lymphocytes.

Moreover, examination of ovaries of high dose female group revealed more abundant fibroblasts with elongated nuclei of fibroblast proliferation and increased number of atretic follicles.

\section{Histopathological results of the testis}

Light microscopic examination for testes of low dose male group revealed that there was failure of spermatogenesis, separation between spermatogenic cells and basement membrane, disruption of some sertoli cells, decreased number of leydig cells (which is responsible for testosterone secretion), deposition of hyaline material between seminefrous tubules and fusion between tubules.

Moreover, examination of testes of high dose male group revealed a more aggressive picture of the previously mentioned changes up to complete degeneration to seminefrous tubules.

\section{Histopathological results of the pituitary glands}


Pituitary gland of the studied female group

Electron microscopic examination showed vacuolated cells (gonadotrophes) with depletion of secretory granules containing LH and FSH while there was no changes in secretory granules containing prolactin that was present in mammotrophic cells. Also, there was some apoptotic gonadotrophic cells with shrunken and piknotic nuclei. Their nuclei showed fragmentation of the nucleus with disrupted nuclear envelope and non apparent nucleolus.

\section{Pituitary gland of the studied male group}

Electron microscopic examination revealed gonadotrophes showing depletion of secretory granules containing LH and FSH also infiltration with lipid droplets were observed while mammotrophic cells were very rich in secretory granules containing prolactin.

Table (1): ANOVA one way statistical analysis comparing female control group (I), low dose female group (II) and high dose female group (III) regarding serum prolactin level.

\begin{tabular}{|c|c|c|c|c|c|}
\hline \multirow{2}{*}{ Prolactin } & \multicolumn{3}{|c|}{ Group } & \multicolumn{2}{c|}{ ANOVA } \\
\cline { 2 - 5 } & Female control group & Low dose female group & High dose female group & F & P-value \\
\hline Range & $5-20.12$ & $8.92-12.3$ & $9.4-12.3$ & \multirow{2}{*}{0.094} & 0.911 \\
\hline Mean \pm SD & $10.751 \pm 4.521$ & $10.787 \pm 1.032$ & $11.142 \pm 0.982$ & \\
\hline
\end{tabular}

I: Female control group; II: Low dose female group; III: High dose female group; F: variance ratio calculated by ANOVA one way statistical analysis; P-value: >0.05: non-significant, <0.05: significant, <0.001: highly significant

Table (2): ANOVA one way statistical analysis and TUKEY'S test comparing female control group (I), low dose female group (II) and high dose female group (III) regarding serum LH level.

\begin{tabular}{|c|c|c|c|c|c|c|c|c|}
\hline \multirow{2}{*}{ LH } & \multicolumn{3}{|c|}{ Group } & \multicolumn{2}{|c|}{ ANOVA } & \multicolumn{3}{|c|}{ TUKEY'S Test } \\
\cline { 2 - 9 } & $\begin{array}{c}\text { Female control } \\
\text { group }\end{array}$ & $\begin{array}{c}\text { Low dose } \\
\text { female group }\end{array}$ & $\begin{array}{c}\text { High dose } \\
\text { female group }\end{array}$ & F & P-value & I\&II & I\&III & II\&III \\
\hline Range & $0.56-1.61$ & $0.31-0.51$ & $0.3-0.47$ & \multirow{2}{*}{50.089} & $<0.001^{*}$ & $<0.001^{*}$ & $<0.001^{*}$ & 0.933 \\
\hline Mean \pm SD & $1.045 \pm 0.347$ & $0.407 \pm 0.063$ & $0.380 \pm 0.053$ & &
\end{tabular}

I: Female control group; II: Low dose female group; III: High dose female group; F: variance ratio calculated by ANOVA one way statistical analysis; P-value: >0.05: non-significant, <0.05: significant, <0.001: highly significant

* There is a highly significant difference between groups by using One Way ANOVA at $p<0.001$.

Table (3): ANOVA one way statistical analysis and TUKEY'S test comparing female control group (I), low dose female group (II) and high dose female group (III) regarding serum FSH level.

\begin{tabular}{|c|c|c|c|c|c|c|c|c|}
\hline \multirow{2}{*}{ FSH } & \multicolumn{4}{|c|}{ Group } & \multicolumn{2}{c|}{ ANOVA } & \multicolumn{3}{|c|}{ TUKEY'S Test } \\
\cline { 2 - 8 } & $\begin{array}{c}\text { Female control } \\
\text { group }\end{array}$ & $\begin{array}{c}\text { Low dose } \\
\text { female group }\end{array}$ & $\begin{array}{c}\text { High dose } \\
\text { female group }\end{array}$ & F & P-value & I\&II & I\&III & II\&III \\
\hline Range & $1.21-1.56$ & $0.31-0.52$ & $0.22-0.35$ & 1176.379 & $<0.001 *$ & $<0.001^{*}$ & $<0.001^{*}$ & $<0.001 *$ \\
\hline Mean \pm SD & $1.320 \pm 0.082$ & $0.415 \pm 0.066$ & $0.283 \pm 0.033$ & &
\end{tabular}

I: Female control group, II: Low dose female group; III: High dose female group; F: variance ratio calculated by ANOVA one way statistical analysis; P-value: $>0.05$ : non-significant, $<0.05$ : significant, $<0.001$ : highly significant

* There is a highly significant difference between groups by using One Way ANOVA at $p<0.001$.

Table (4): ANOVA one way statistical analysis and TUKEY'S test comparing female control group (I), low dose female group (II) and high dose female group (III) regarding serum Estradiol (E2) level.

\begin{tabular}{|c|c|c|c|c|c|c|c|c|}
\hline \multirow{2}{*}{ Estradiol } & \multicolumn{3}{|c|}{ Group } & \multicolumn{2}{c|}{ ANOVA } & \multicolumn{3}{|c|}{ TUKEY'S Test } \\
\cline { 2 - 9 } & $\begin{array}{c}\text { Female control } \\
\text { group }\end{array}$ & $\begin{array}{c}\text { Low dose } \\
\text { female group }\end{array}$ & $\begin{array}{c}\text { High dose } \\
\text { female group }\end{array}$ & F & P-value & I\&II & I\&III & II\&III \\
\hline Range & $35.25-48.02$ & $26.97-28.35$ & $16.13-22.02$ & \multirow{2}{*}{380.161} & $<0.001^{*}$ & $<0.001^{*}$ & $<0.001^{*}$ & $<0.001^{*}$ \\
\hline Mean \pm SD & $42.565 \pm 3.438$ & $27.613 \pm 0.481$ & $20.172 \pm 1.829$ & &
\end{tabular}

I: Female control group, II: Low dose female group; III: High dose female group; F: variance ratio calculated by ANOVA one way statistical analysis; $P$-value: $>0.05$ : non-significant, $<0.05$ : significant, $<0.001$ : highly significant

* There is a highly significant difference between groups by using One Way ANOVA at $p<0.001$. 
Table (1): ANOVA one way statistical analysis and TUKEY'S test comparing female control group (I), low dose female group (II) and high dose female group (III) regarding serum progesterone level.

\begin{tabular}{|c|c|c|c|c|c|c|c|c|}
\hline \multirow{2}{*}{ Progesteron } & \multicolumn{3}{|c|}{ Group } & \multicolumn{2}{c|}{ ANOVA } & \multicolumn{3}{|c|}{ TUKEY'S Test } \\
\cline { 2 - 9 } & $\begin{array}{c}\text { Female control } \\
\text { group }\end{array}$ & $\begin{array}{c}\text { Low dose } \\
\text { female group }\end{array}$ & $\begin{array}{c}\text { High dose } \\
\text { female group }\end{array}$ & F & P-value & I\&II & I\&III & II\&III \\
\hline Range & $0.27-0.34$ & $0.64-1.1$ & $0.19-0.28$ & 337.611 & $<0.001^{*}$ & $<0.001^{*}$ & $0.040^{*}$ & $<0.001^{*}$ \\
\hline Mean \pm SD & $0.293 \pm 0.019$ & $0.800 \pm 0.109$ & $0.232 \pm 0.027$ & 337.619 &
\end{tabular}

I: Female control group, II: Low dose female group; III: High dose female group; F: variance ratio calculated by ANOVA one way statistical analysis; P-value: $>0.05$ : non-significant, $<0.05$ : significant, $<0.001$ : highly significant

* There is a highly significant difference between groups by using One Way ANOVA at $p<0.001$.

Table (2): ANOVA one way statistical analysis and TUKEY'S test comparing female control group (I), low dose female group (II) and high dose female group (III) regarding weight before and after the experiment.

\begin{tabular}{|c|c|c|c|c|c|c|c|c|c|}
\hline \multirow{2}{*}{\multicolumn{2}{|c|}{ Weight }} & \multicolumn{3}{|c|}{ Group } & \multicolumn{2}{|c|}{ ANOVA } & \multicolumn{3}{|c|}{ TUKEY'S Test } \\
\hline & & $\begin{array}{l}\text { Female control } \\
\text { group }\end{array}$ & $\begin{array}{c}\text { Low dose } \\
\text { female group }\end{array}$ & $\begin{array}{c}\text { High dose } \\
\text { female group }\end{array}$ & $\mathbf{F}$ & P-value & I\&II & I\&III & II\&III \\
\hline \multirow{2}{*}{ Before } & Range & 133-155 & $136-151$ & $138-147$ & \multirow{2}{*}{0.006} & \multirow{2}{*}{0.994} & & & \\
\hline & Mean \pm SD & $143.067 \pm 6.819$ & $143.000 \pm 4.781$ & $142.867 \pm 3.091$ & & & & & \\
\hline \multirow{2}{*}{ After } & \begin{tabular}{|l|} 
Range \\
\end{tabular} & 161-183 & $165-183$ & 154-171 & \multirow{2}{*}{11.388} & \multirow{2}{*}{$<0.001^{*}$} & \multirow{2}{*}{0.337} & \multirow{2}{*}{$<0.001^{*}$} & \multirow{2}{*}{$0.007^{*}$} \\
\hline & Mean \pm SD & $174.733 \pm 7.086$ & $171.733 \pm 4.803$ & $164.933 \pm 5.133$ & & & & & \\
\hline Differences & Mean \pm SD & $-31.667 \pm 4.203$ & $-28.733 \pm 3.173$ & $-22.067 \pm 5.535$ & & & & & \\
\hline Paired test & P-value & $<0.001 *$ & $<0.001^{*}$ & $<0.001^{*}$ & & & & & \\
\hline
\end{tabular}

I: Female control group, II: Low dose female group; III: High dose female group; F: variance ratio calculated by ANOVA one way statistical analysis; P-value: $>0.05$ : non-significant, $<0.05$ : significant, $<0.001$ : highly significant

* There is a highly significant difference between groups by using One Way ANOVA at $p<0.001$.

Table (3): ANOVA one way statistical analysis and TUKEY'S test comparing male control group (I), low dose male group (II) and high dose male group (III) regarding serum prolactin level.

\begin{tabular}{|c|c|c|c|c|c|c|c|c|}
\hline \multirow{2}{*}{ Prolactin } & \multicolumn{3}{|c|}{ Group } & \multicolumn{2}{|c|}{ ANOVA } & \multicolumn{3}{|c|}{ TUKEY'S Test } \\
\cline { 2 - 9 } & $\begin{array}{c}\text { Male control } \\
\text { group }\end{array}$ & $\begin{array}{c}\text { Low dose } \\
\text { male group }\end{array}$ & $\begin{array}{c}\text { High dose } \\
\text { male group }\end{array}$ & F & P-value & I\&II & I\&III & II\&III \\
\hline Range & $4.72-10.4$ & $9.87-14.09$ & $10.7-12.5$ & 62.712 & $<0.001^{*}$ & $<0.001^{*}$ & $<0.001^{*}$ & 0.988 \\
\hline Mean \pm SD & $7.502 \pm 1.482$ & $11.551 \pm 1.141$ & $11.489 \pm 0.602$ & 62.712 \\
\hline
\end{tabular}

I: Female control group, II: Low dose female group; III: High dose female group; F: variance ratio calculated by ANOVA one way statistical analysis; P-value: $>0.05$ : non-significant, $<0.05$ : significant, $<0.001$ : highly significant

* There is a highly significant difference between groups by using One Way ANOVA at $p<0.001$.

Table (4): ANOVA one way statistical analysis and TUKEY'S test comparing male control group (I), low dose male group (II) and high dose male group (III) regarding serum LH level.

\begin{tabular}{|c|c|c|c|c|c|c|c|c|}
\hline \multirow{2}{*}{ LH } & \multicolumn{3}{|c|}{ Group } & \multicolumn{2}{c|}{ ANOVA } & \multicolumn{3}{c|}{ TUKEY'S Test } \\
\cline { 2 - 9 } & $\begin{array}{c}\text { Male control } \\
\text { group }\end{array}$ & $\begin{array}{c}\text { Low dose } \\
\text { male group }\end{array}$ & $\begin{array}{c}\text { High dose } \\
\text { male group }\end{array}$ & F & P-value & I\&II & I\&III & II\&III \\
\hline Range & $3.4-5.67$ & $0.69-0.93$ & $0.35-0.77$ & 479.003 & $<0.001^{*}$ & $<0.001^{*}$ & $<0.001^{*}$ & 0.247 \\
\hline Mean \pm SD & $4.624 \pm 0.687$ & $0.799 \pm 0.072$ & $0.559 \pm 0.109$ & 49.047 \\
\hline
\end{tabular}

I: Female control group, II: Low dose female group; III: High dose female group; F: variance ratio calculated by ANOVA one way statistical analysis; P-value: $>0.05$ : non-significant, $<0.05$ : significant, $<0.001$ : highly significant

* There is a highly significant difference between groups by using One Way ANOVA at $p<0.001$.

Table (5): ANOVA one way statistical analysis and TUKEY'S test comparing male control group (I), low dose male group (II) and high dose male group (III) regarding serum FSH level.

\begin{tabular}{|c|c|c|c|c|c|c|c|c|}
\hline \multirow{2}{*}{ FSH } & \multicolumn{3}{|c|}{ Group } & \multicolumn{2}{c|}{ ANOVA } & \multicolumn{3}{|c|}{ TUKEY'S Test } \\
\cline { 2 - 9 } & $\begin{array}{c}\text { Male control } \\
\text { group }\end{array}$ & $\begin{array}{c}\text { Low dose } \\
\text { male group }\end{array}$ & $\begin{array}{c}\text { High dose } \\
\text { male group }\end{array}$ & F & P-value & I\&II & I\&III & II\&III \\
\hline Range & $1.55-1.74$ & $0.38-0.55$ & $0.22-0.37$ & 3420.766 & $<0.001^{*}$ & $<0.001^{*}$ & $<0.001^{*}$ & $<0.001^{*}$ \\
\hline Mean \pm SD & $1.641 \pm 0.053$ & $0.473 \pm 0.053$ & $0.306 \pm 0.038$ & &
\end{tabular}

I: Female control group, II: Low dose female group; III: High dose female group; F: variance ratio calculated by ANOVA one way statistical analysis; P-value: >0.05: non-significant, <0.05: significant, $<0.001$ : highly significant

* There is a highly significant difference between groups by using One Way ANOVA at $p<0.001$. 
Table (6): ANOVA one way statistical analysis and TUKEY'S test comparing male control group (I), low dose male group (II) and high dose male group (III) regarding serum Estradiol (E2) level.

\begin{tabular}{|c|c|c|c|c|c|c|c|c|}
\hline \multirow{2}{*}{ Estradiol } & \multicolumn{4}{|c|}{ Group } & \multicolumn{2}{|c|}{ ANOVA } & \multicolumn{3}{|c|}{ TUKEY'S Test } \\
\cline { 2 - 9 } & $\begin{array}{c}\text { Male control } \\
\text { group }\end{array}$ & $\begin{array}{c}\text { Low dose } \\
\text { male group }\end{array}$ & $\begin{array}{c}\text { High dose } \\
\text { male group }\end{array}$ & F & P-value & I\&II & I\&III & II\&III \\
\hline Range & $18.28-21.84$ & $13.33-22.7$ & $15.33-19.7$ & 4.305 & $0.020^{*}$ & 0.990 & $0.033^{*}$ & $0.046^{*}$ \\
\hline Mean \pm SD & $19.822 \pm 0.904$ & $19.744 \pm 2.313$ & $18.342 \pm 1.041$ & 4.305 &
\end{tabular}

I: Female control group, II: Low dose female group; III: High dose female group; F: variance ratio calculated by ANOVA one way statistical analysis; P-value: $>0.05$ : non-significant, $<0.05$ : significant, $<0.001$ : highly significant

* There is a highly significant difference between groups by using One Way ANOVA at $p<0.001$.

Table (7): ANOVA one way statistical analysis and TUKEY'S test comparing male control group (I), low dose male group (II) and high dose male group (III) regarding serum testosterone level.

\begin{tabular}{|c|c|c|c|c|c|c|c|c|}
\hline \multirow{2}{*}{ Testosterone } & \multicolumn{4}{|c|}{ Group } & \multicolumn{2}{|c|}{ ANOVA } & \multicolumn{3}{|c|}{ TUKEY'S Test } \\
\cline { 2 - 9 } & $\begin{array}{c}\text { Male control } \\
\text { group }\end{array}$ & $\begin{array}{c}\text { Low dose } \\
\text { male group }\end{array}$ & $\begin{array}{c}\text { High dose } \\
\text { male group }\end{array}$ & F & P-value & I\&II & I\&III & II\&III \\
\hline Range & $7.05-9.47$ & $7.12-9.75$ & $6.8-7.67$ & 19.738 & $<0.001^{*}$ & 0.717 & $<0.001^{*}$ & $<0.001^{*}$ \\
\hline Mean \pm SD & $8.461 \pm 0.730$ & $8.652 \pm 0.871$ & $7.233 \pm 0.249$ & 19.73 &
\end{tabular}

I: Female control group, II: Low dose female group; III: High dose female group; F: variance ratio calculated by ANOVA one way statistical analysis; P-value: $>0.05$ : non-significant, <0.05: significant, $<0.001$ : highly significant

* There is a highly significant difference between groups by using One Way ANOVA at $p<0.001$.

Table (8): ANOVA one way statistical analysis and TUKEY'S test comparing male control group (I), low dose male group (II) and high dose male group (III) regarding weight before and after the experiment.

\begin{tabular}{|c|c|c|c|c|c|c|c|c|c|}
\hline \multirow{2}{*}{\multicolumn{2}{|c|}{ Weight }} & \multicolumn{3}{|c|}{ Group } & \multicolumn{2}{|c|}{ ANOVA } & \multicolumn{3}{|c|}{ TUKEY'S Test } \\
\hline & & $\begin{array}{l}\text { Male control } \\
\text { group }\end{array}$ & $\begin{array}{l}\text { Low dose } \\
\text { male group }\end{array}$ & $\begin{array}{l}\text { High dose } \\
\text { male group }\end{array}$ & $\mathbf{F}$ & P-value & I\&II & I\&III & II\&III \\
\hline \multirow{2}{*}{ Before } & \begin{tabular}{|l|} 
Range \\
\end{tabular} & $146-157$ & $146-158$ & $150-158$ & \multirow{2}{*}{2.034} & \multirow{2}{*}{0.144} & & & \\
\hline & Mean \pm SD & $151.867 \pm 3.067$ & $151.933 \pm 3.390$ & $153.800 \pm 2.396$ & & & & & \\
\hline \multirow{2}{*}{ After } & Range & $186-223$ & $182-194$ & $167-173$ & \multirow{2}{*}{74.969} & \multirow{2}{*}{$<0.001 *$} & \multirow{2}{*}{$<0.001 *$} & \multirow{2}{*}{$<0.001^{*}$} & \multirow{2}{*}{$<0.001^{*}$} \\
\hline & Mean \pm SD & $201.667 \pm 11.011$ & $187.400 \pm 4.102$ & $171.000 \pm 1.813$ & & & & & \\
\hline Differences & Mean \pm SD & $-49.800 \pm 10.805$ & $-35.467 \pm 1.959$ & $-17.200 \pm 1.859$ & & & & & \\
\hline Paired test & P-value & $<0.001^{*}$ & $<0.001 *$ & $<0.001 *$ & & & & & \\
\hline
\end{tabular}

I: Female control group, II: Low dose female group; III: High dose female group; F: variance ratio calculated by ANOVA one way statistical analysis; P-value: $>0.05$ : non-significant, <0.05: significant, $<0.001$ : highly significant

* There is a highly significant difference between groups by using One Way ANOVA at $p<0.001$.

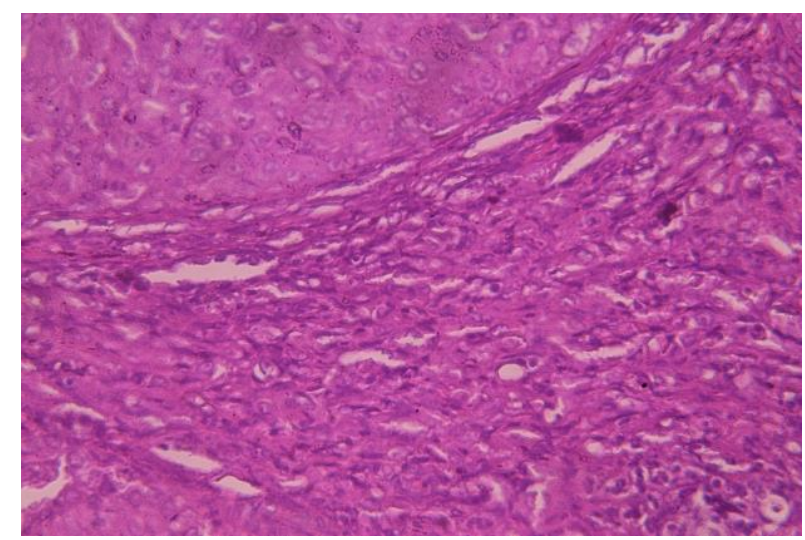

Figure (1): Photomicrograph of adult albino rat ovary of low dose female group showing abundant fibroblasts between follicles $(\mathrm{H} \& \mathrm{E} \times 40)$.

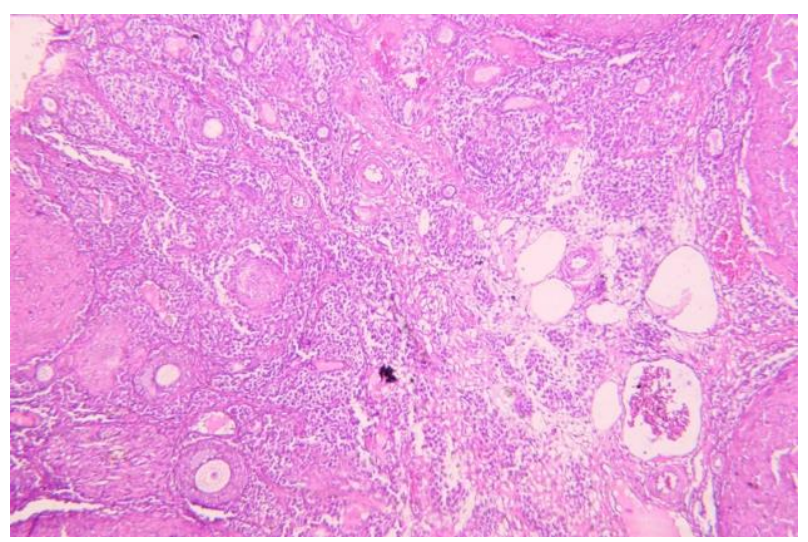

Figure (2): Photomicrograph of adult albino rat ovary of high dose female group showing multiple atretic follicles $(\mathrm{H} \& \mathrm{E} \times \mathbf{1 0})$. 


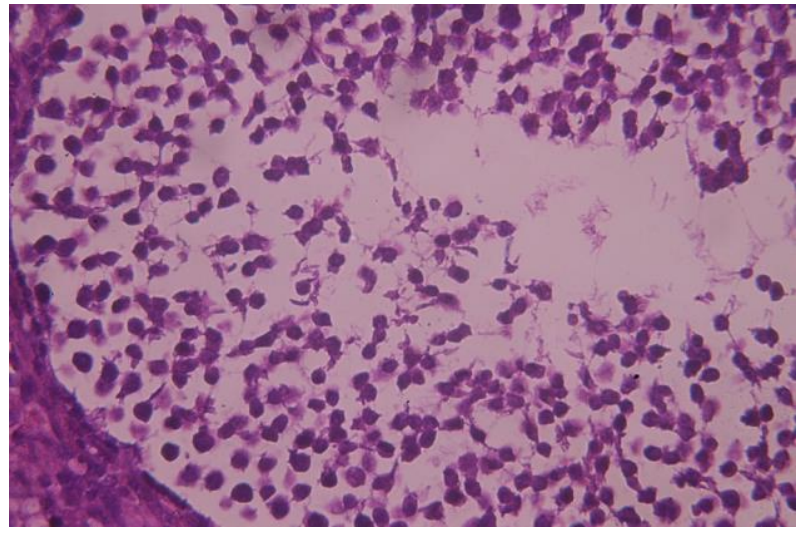

Figure (3): Photomicrograph of adult albino rat testis of low dose male group showing failure of spermatogenesis, no sperms inside tubule $(\mathrm{H} \& \mathrm{E} \times 10)$.

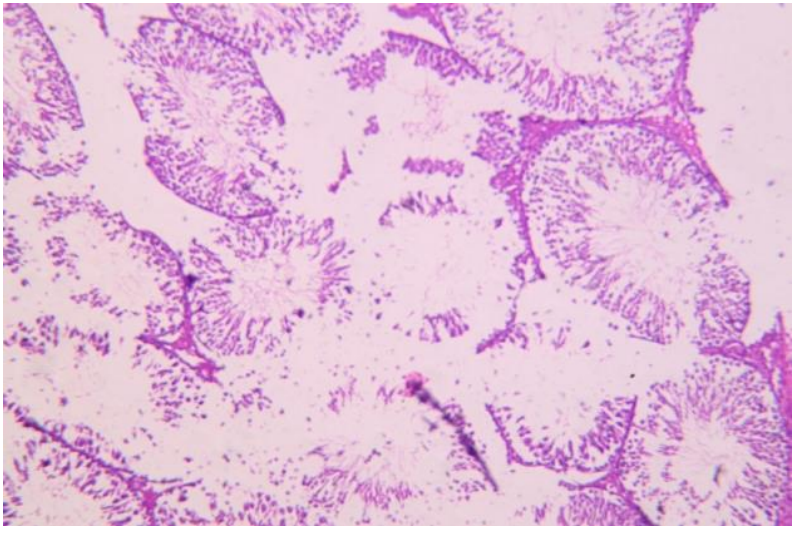

Figure (4): Photomicrograph of adult albino rat testis of high dose male group showing massive degeneration of seminiferous tubules, rupture of basement membrane and failure of spermatogenesis $(\mathrm{H} \& \mathrm{E} \times 10)$.

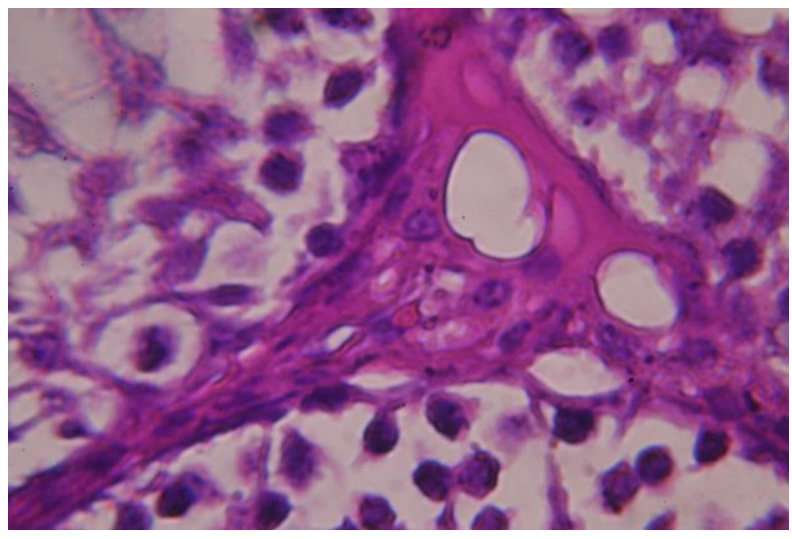

Figure (5): Photomicrograph of adult albino rat testis of high dose male group showing decreased number of leydig cells between tubules $(\mathrm{H} \& \mathrm{E} \times \mathbf{1 0})$.

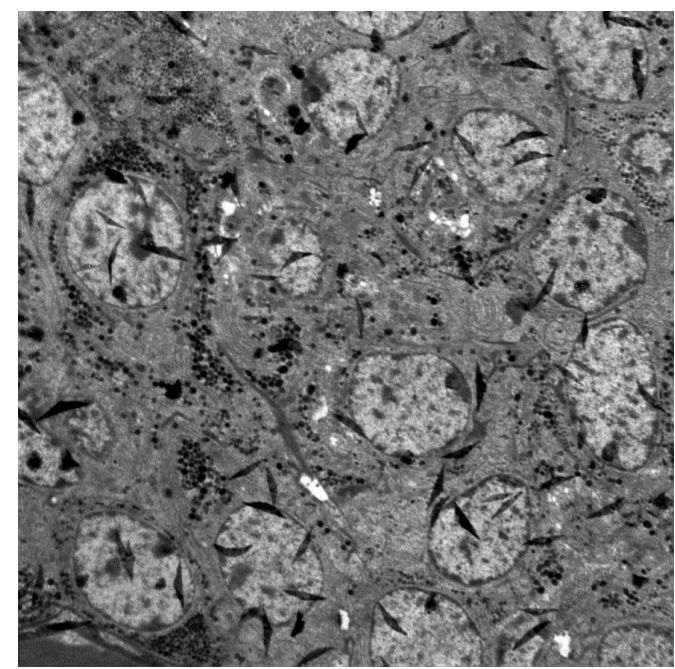

$\overline{2 \text { microns }}$

Figure (6): Electron microscopic image of adult albino rat pituitary gland of low dose female group showing gonadotrophes with vacuolated cells with depletion of granules.

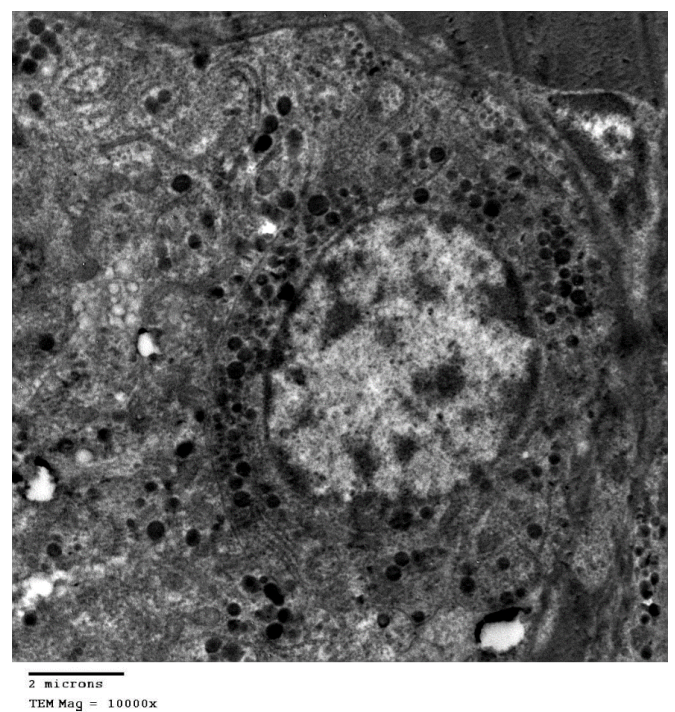

Figure (7): Electron microscopic image of adult albino rat pituitary gland of high dose female group showing gonadotrophes with vacuolated cells together with normal mammotrophes. 


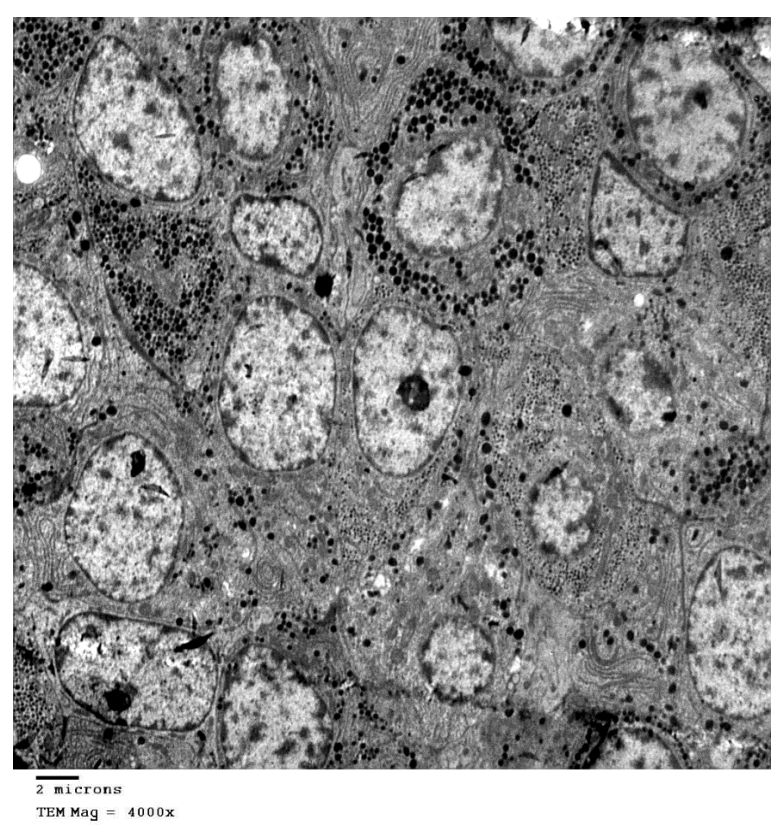

Figure (8): Electron microscopic image of adult albino rat pituitary gland of low dose male group showing gonadotrophes with depletion of secretory granules, a mmamotrophe also appears at the same section that is rich in secretory granules.

\section{Discussion}

Chronic opioid use may predispose to hypogonadism through alteration of the hypothalamic pituitary-gonadal axis. Opioids seem to mediate their effects on gonadal hormones primarily via central mechanisms (acting on the hypothalamus and pituitary), although a peripheral component through direct effect on target organs may be involved as well (Gudin et al., 2015). The present experimental study evaluated the effect of chronic tramadol use on the reproductive hormones and the related pathological changes.

Regarding the hormones levels in the female rats groups in the current study, there was non-significant difference in serum prolactin levels between tramadol treated groups (low dose and high dose) and the control group. This was confirmed by histological examination of the pituitary glands belonging to the same groups where there was no abnormality in the cells secreting prolactin (mammotrophes). Contrary to the result of the current study, Roberts et al. (2002) reported opioid suppression of the HPG (hypothalamo pituitary gonadal) axis in women resulting in amenorrhea in heroin addicts.

As regard serum leutinizing hormone (LH) levels, the current study revealed that there was a significant decrease among tramadol treated female groups whether receiving low or high dose compared to the control group. This was confirmed by histological examination of ovaries belonging to the same groups where the stroma showed abundant increase of fibroblast activity which correlated with the observed decrease in serum LH level. These findings are in accordance with

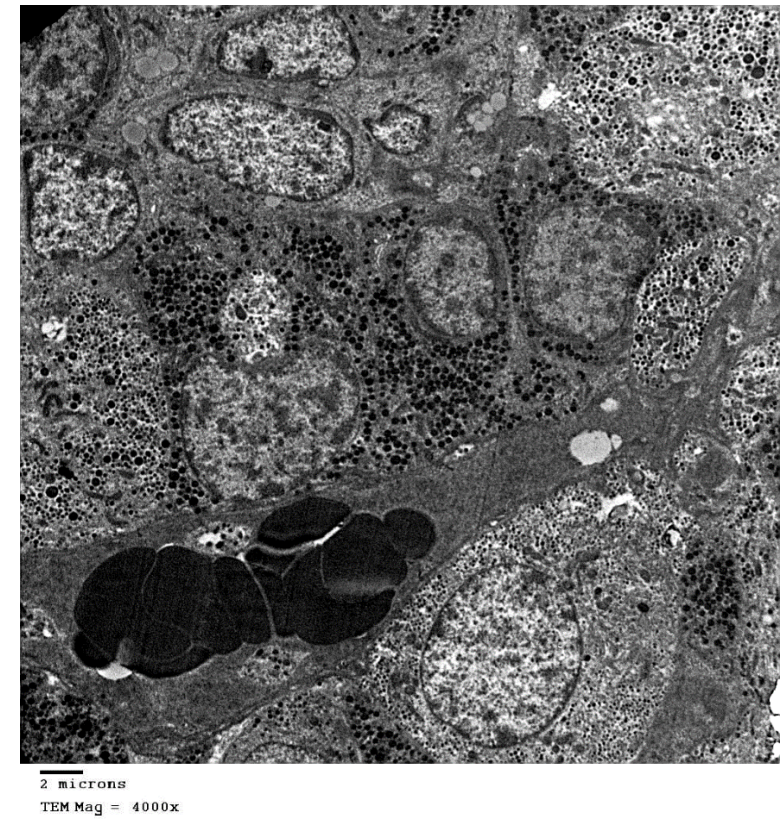

Figure (9): Electron microscopic image of adult albino rat pituitary gland of high dose male group showing mammotrophic cell that is rich in secretory granules.

the findings obtained by Vuong et al. (2009) who mentioned that chronic opioid administration decreases LH levels in both animals and humans, and that the effect on LH occurs primarily by inhibiting hypothalamic gonadotrophin releasing hormone $(\mathrm{GnRH})$ secretion.

By studying the effect of tramadol on serum follicular stimulating hormone (FSH) levels in the present study, it showed a statistically significant decrease among tramadol treated female groups whether receiving low or high dose compared to the control group. In addition, the high dose treated group showed a statistically significant decrease in serum FSH when compared to the low dose treated group. This was reflected on the histopathological changes in the ovaries of the same groups which showed a detected damage of the growing follicles. Many of the mature follicles appeared atretic in the form of cystic-like structures.

These finding are in accordance with a study conducted by Daniell (2006) which showed that women consuming sustained-action oral or transdermal opioids for the control of noncancer pain have a decrease in FSH and LH serum levels. Furthermore, Youssef and Zidan (2016) stated that tramadol administration caused a significant decrease in serum levels of LH and FSH.

The histological findings of the current study are similar to those obtained by El-Ghawet (2015) who observed that in experimental tramadol-treatment, there was a detected damage of the growing follicles. They observed that many of the mature follicles appeared atretic in the form of cystic-like structures. Atretic 
follicles possessed granulosa cells with the apparent increase of pyknosis and deformed antral cavity, and the stroma showed abundant increase of fibroblast activity.

Tramadol not only acts centrally affecting reproductive hormonal release, but also acts peripherally affecting the production of sex steroids (Katz and Mazer, 2009). The present study revealed that there was a statistical significant decrease in serum estradiol among tramadol treated female groups when compared with the control group. In addition, when comparing the low dose and high dose groups, the high dose treated group showed statistically significant decrease in serum estradiol when compared with the low dose treated group.

Vuong et al. (2009) mentioned that chronic opioid administration results in suppression of sex steroids mediated mainly by suppression of $\mathrm{LH}$ via the effects on GnRH. In addition, there may be direct effects at the gonadal level. In addition, a study conducted by Daniell (2006) showed that women consuming sustainedaction oral or transdermal opioids for the control of noncancerous pain have a profound inhibition of ovarian sex hormones namely progesterone and estrogen.

Regarding serum progesterone level, the present study revealed that there was a statistically significant decrease among the high dose tramadol treated female group when compared to both the low dose tramadol treated group and the control group but there was a statistically significant increase when comparing the low dose to the control group and the high dose group. This fluctuation in serum progesterone level explains the irregularities in menstruation that may be associated with tramadol intake.

In a study by Abs et al. (2002) they found that exogenous opioids have a drastic effect on the female menstrual cycle. In their study, after long-term intrathecal opioid administration, 14 of 21 premenopausal women developed amenorrhea, and the other seven developed irregularities in menstruation. Opioids led to a decline in LH, FSH, estradiol, and progesterone, thus affecting menstruation.

Regarding weight changes, the current study showed that there was a statistically significant decrease in weight in the high dose treated group when compared to both the low dose treated group and the control group. Similar findings were observed by Matthiesen et al. (1998) who stated that there was a decrease of body weight gain when female rats were treated for 14 days with $50 \mathrm{mg} / \mathrm{kg}$ tramadol.

Regarding the male groups, the current study revealed that serum prolactin (PRL) level showed a statistically significant increase in both the low dose and the high dose groups when compared to the control group. This was supported by the histopathological findings while examining the pituitary glands that belong to the same groups where abundant and hypertrophied mammotrophes were seen.
This goes hand in hand with the findings of $E$ gaafarawi (2006) who stated that administration of 40 $\mathrm{mg}$ tramadol $/ \mathrm{Kg}$ in rats led to statistically elevated serum prolactin level at 20 and 30 days of treatment. In addition, a study by Youssef and Zidan (2016) revealed that there was a statistically significant increase in serum prolactin (PRL) level among tramadol receiving rat groups when compared to control group. This effect appears to be mediated by hypothalamic factors rather than via direct action on the pituitary.

By studying the effect of tramadol on serum LH level among male groups in the current study, it showed a statistically significant decrease level in both treated male groups (low dose and high dose) as compared to the control group. The histological examination of testes of those groups revealed that seminiferous tubules were lacking sperms, spermatids and secondary spermatocytes. Exfoliation of the damaged spermatocytes and spermatids were detected within the tubular lumina of many seminiferous tubules. The intertubular connective tissue became hyalinized and showed comparative reduction of interstitial cells.

A study by Elliott et al. (2011) documented hypogonadism with decreased LH/FSH levels in both males and females as early as 1 week after starting opioid therapy, with LH levels more affected in males.

By studying the effect of tramadol on serum FSH level in tramadol treated male groups, there was a statistically significant decrease in serum FSH level in both low dose and the high dose tramadol treated male groups as compared with control group. This was supported by the histological findings of testicular examination among tramadol treated groups where a failure of spermatogenesis was recorded in addition to separation between spermatogonic cells and the basement membrane and disruption of some sertoli cells.

These findings were in accordance to a study by Roberts et al. (2002) who mentioned that intrathecal opioid therapy in men results in suppression of the hypothalamo-pituitary gonadal (HPG) axis as there was a statistically significant decrease in FSH levels and a decline in serum testosterone levels. They explained that this is a central effect because direct suppression at a testicular level would result in a secondary increase in gonadotrophin (FSH and LH) levels.

In addition, A study by Rhodin et al. (2010) compared 39 chronic pain patients treated with opioid agonists for more than 12 months to a 20 pain patients not on opioids. In comparing the two groups, lower values of LH and FSH were observed among the opioid group. These low values of $\mathrm{LH}$ and FSH suggest an inhibitory effect of opioids on the hypothalamic-pituitary levels.

The histological findings of the current study are similar the findings observed by a study by Youssef and Zidan (2016) that showed atrophy of seminiferous tubules with interstitial calcification and focal testicular 
degeneration with single or multiple layers of vacuolated spermatocytes with a little evidence of spermatogenesis.

Regarding serum estradiol (E2) level among the male groups in the current study, a statistically significant decrease was observed in the high dose tramadol treated group when compared to both the control group and low dose treated group, while there was a nonsignificant difference between the control group and the low dose treated group. This goes hand in hand with a study by Yilmaz (1999) that mentioned that morphine administration resulted in decrease in $\mathrm{LH}$ levels and estradiol levels as well.

According to the present study, testosterone level showed a statistically significant decrease among high dose tramadol treated male group when compared to both the control and low dose treated groups, while there was a nonsignificant level difference between both the control and the low dose treated male groups. This is supported by a study by Roberts et al (2002) where they observed that intrathecal opioid therapy in men resulted in suppression of the hypothalamo-pituitary gonadal (HPG) axis and a decline in serum testosterone levels.

Regarding weight changes among male groups, the current study showed statistically significant decrease in weight gain among male treated groups both the low dose and the high dose when compared to the control group. These finding matched the findings of a study by Anghel et al. (2010) that observed the effects of shortand long-term morphine administration on rodent body weight where they found a decrease in food intake coupled with marked weight loss in the days after morphine treatment.

\section{Conclusion}

From the current study it was concluded that:

Chronic use of tramadol in rats even with its therapeutic doses has bad effects on gonadal function affecting both males and females. This is caused by the inhibitory effects of tramadol on the production of gonadal hormones which was observed in the current study through low levels of LH, FSH and Estradiol in both males and females and low testosterone level among males and low progesterone level among females. Also, hyperprolactinemia was observed among tramadol treated male groups while serum prolactin level was not affected among females.

These hormonal changes were due to structural changes affecting tissues. So histopathological examination of the pituitary glands of both males and females adult albino rats under the electron microscope revealed apoptosis of gonadotrophes (secreting LH and FSH) with depletion of secretory granules. While there was hypertrophy of mammotrophes (secreting prolactin) with abundant granules and this was observed among tramadol treated male groups but not among female groups. On the other hand, light microscopic examination for ovaries of tramadol treated female groups revealed abundant fibroblasts with elongated nuclei of fibroblast proliferation, atretic follicles, congestion between the follicles and aggregation of lymphocytes. In addition, light microscopic examination for testes of tramadol treated male groups revealed failure of spermatogenesis, separation between spermatogenic cells and basement membrane, disruption of some sertoli cells, decrease number of leydig cells (which is responsible for testosterone secretion), deposition of hyaline material between seminefrous tubules and fusion between tubules.

\section{Recommendations}

- The present study recommends that further studies on the reproductive effects of tramadol should be done, with focus on the effects on females as most studies used to focus on the effects on males.

- More studies should examine the histopathological effects of tramadol especially on pituitary glands and related hormonal changes as not only gonadotrophic hormones are expected to be affected but also the other pituitary hormones as well.

- Human studies on tramadol users regarding the effects of tramadol on reproductive hormones should be performed to enhance our evidence upon its reproductive effects and to assess the hormonal levels after discontinuation of the drug.

\section{References}

Abs R., Verhelst J., Maeyaert J., Van Buyten J. P., Opsomer F., Adriaensen H., Verlooy J., Van Havenbergh T., Smet M., Van Acker K. (2002): Endocrine consequences of long-term intrathecal administration of opioids. The Journal of Clinical Endocrinology \& Metabolism. 85(6): 2215-22.

Anghel A., Jamieson C. A., Ren X., Young J., Porche R., Ozigbo E., Ghods D. E., Lee M. L., Liu Y., Lutfy K., Friedman T. C. (2010): Gene expression profiling following short-term and long-term morphine exposure in mice uncovers genes involved in food intake. Neuroscience. 167(2): 554-66.

Bassiony M. M., Salah El-Deen G. M., Yousef U., Raya Y., Abdel-Ghani M. M., El-Gohari H., Atwa S. A. (2015): Adolescent tramadol use and abuse in Egypt. The American Journal of Drug and Alcohol Abuse. 41(3): 206-11.

Daniell H. W. (2002): Hypogonadism in men consuming sustained-action oral opioids. The Journal of Pain. 3(5): 377-84.

Daniell H. W. (2006): DHEAS deficiency during consumption of sustained-action prescribed opioids: evidence for opioid-induced inhibition of adrenal androgen production. The Journal of Pain. 7(12): 901-7. 
Drury R. A B., Wallington E. A. (1980): Preparation and fixation of tissues. In: Carleton's histological technique. Fifth Edition. 41-54.

Egan T. D., Newberry C. (2018): Opioids. In: Basics of Anesthesia E-Book. Seventh Edition. Elsevier Inc. 123-138.

El-Gaafarawi I. I. (2006): Biochemical toxicity induced by tramadol administration in male rats. Egypt $\mathrm{J}$ Hosp Med. 23(1): 353-62.

El-Ghawet H. A. (2015): Effects of tramadol on the reproductive function of wistar albino rats. Eur $\mathrm{J}$ Exp Biol. 5(1): 56-64.

Elliott J. A., Horton E., Fibuch E. E. (2011): The endocrine effects of long-term oral opioid therapy: A case report and review of the literature. J Opioid Manag. 7(2): 145-154.

Gudin J. A., Laitman A., Nalamachu S. (2015): Opioid related endocrinopathy. Pain Medicine. 16(1): 915.

Gudin J. A., Laitman A., Nalamachu S. (2015): Opioid related endocrinopathy. Pain Medicine. 16(1): 915.

Katz N., Mazer N. A. (2009): The impact of opioids on the endocrine system. The Clinical Journal of Pain. 25(2): 170-5.

Luft J. H. (1961): Improvements in epoxy resin embedding methods. The Journal of Biophysical and Biochemical Cytology. 9 (2): 409.

Manchikanti L., Damron K. S., McManus C. D., Barnhill R. C. (2004): Patterns of illicit drug use and opioid abuse in patients with chronic pain at initial evaluation: a prospective, observational study. Pain Physician. 7(4): 431-7.

Matthiesen T., Wöhrmann T., Coogan T. P., Uragg H. (1998): The experimental toxicology of tramadol: an overview. Toxicology Letters. 95(1):63-71.

Pasternak G. W. (2001): Incomplete cross tolerance and multiple mu opioid peptide receptors. Trends in Pharmacological Sciences. 22(2): 67-70.
Rhodin A., Stridsberg M., Gordh T. (2010): Opioid endocrinopathy: a clinical problem in patients with chronic pain and long-term oral opioid treatment. The Clinical journal of pain. 26(5): 374-380.

Roberts L. J., Finch P. M., Pullan P. T., Bhagat C. I., Price L. M. (2002): Sex hormone suppression by intrathecal opioids: a prospective study. The Clinical Journal of Pain. 18(3): 144-8.

Senay E. C., Adams E. H., Geller A., Inciardi J. A., Munoz A., Schnoll S. H., Woody G. E., Cicero T. J. (2003): Physical dependence on Ultram ${ }^{\circledR}$ (tramadol hydrochloride): both opioidlike and atypical withdrawal symptoms occur. Drug and Alcohol Dependence. 69(3): 233-41.

Trescot AM, Boswell MV, Atluri SL, Hansen HC, Deer TR, Abdi S, Jasper JF, Singh V, Trescot A. M., Boswell M. V., Atluri S. L., Hansen H. C., Deer T. R., Abdi S., Jasper J. F., Singh V., Jordan A. E., Johnson B. W., Cicala R. S. (2006): Opioid guidelines in the management of chronic noncancer pain. Pain Physician. 9(1): 1-40.

Vuong C., Van Uum S. H., O'Dell L. E., Lutfy K., Friedman T. C. (2009): The effects of opioids and opioid analogs on animal and human endocrine systems. Endocrine Reviews. 31(1): 98-132.

Yilmaz B., Knoar V., Kutlu S. (1999): Influence of chronic morphine exposure on serum LH, FSH, testosterone levels, and body and testicular weights in the developing male rat. Archives of andrology. 43(3): 189-196.

Youssef H., Zidan A. (2016): Histopathological and biochemical effects of acute \& chronic tramadol drug toxicity on liver, kidney and testicular function in adult male albino rats. Journal of Medical Toxicology and Clinical Forensic Medicine. 2: 1-7. 


\section{تأثير تناول الترامادول المزمن على الهرمونات التناسلية والتغيرات النسيجيه ذات الصلة في الفئران البيضاء البالغة}

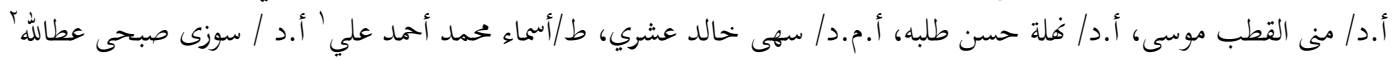

\section{الملخص العربيى}

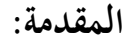

الخلفية: يعتبر الترامادول من المورفينات المصنعه كمسكن للألام. والذي يستخدم على نطاق واسع في علاج خشونة المفاصل وألام الظهر والأصابات الرياضيه والحالات الروماتيزميه والصداع. فهو عقار شائع وصفه لتخفيف الألام حيث أنه أكثر أمانا من المسكنات المورفينيه. الهدف: دراسة تأثير تناول الترامادول المزمن على الهرمونات التناسلية والتغيرات النسيجيه ذات الصلة على المبايض والخصى والغدد النخاميه في الفئران البيضاء البالغة

الطرق: تم إجراء هذه الدراسة التجريبة على تسعون (•9) من الفئران البيضاء البالغة الذكور و الإناث. تم تقسيمهم إلى ثلاث بحموعات تضم كل منها . ب فأر (10 ذكور وه1 إناث). البحموعة الأولى كانت البمموعة الضابطة التي تتلقى محلول ملحي طبيعي، البحموعة الثانية

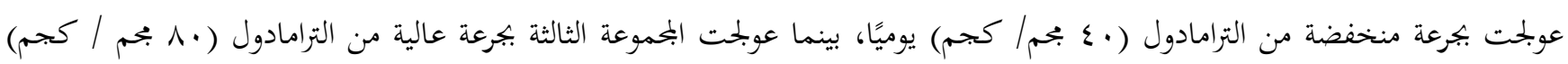
يوميًا. واستمر اعطاء الدواء يوميا لمدة شهر.

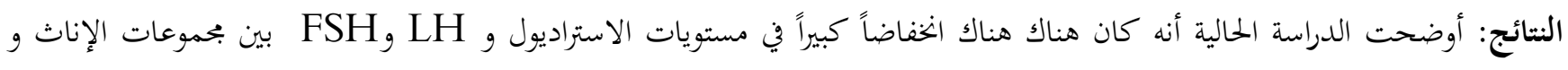
الذكور المعالجة بالترامادول مقارنةً بمجموعة المراقبة. كما أنه كان هناك الخفاض ملحوظ في مستوى هرمون التستوستيرون بين بحموعات الذكور

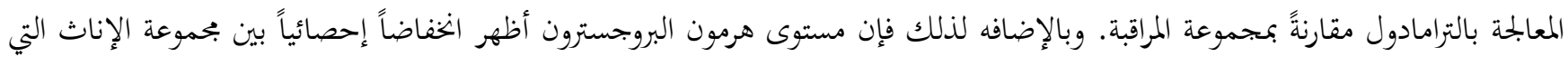
عولحت بجرعة عالية من الترامادول عند مقارنتها بكل من البمموعة التي عولجت بجرعة منخفضة من الترامادول وبحموعة المراقبه ولكن كان هناك زياده في مستوى الهرمون عند مقارنة المحموعات المعالجة بالجرعات المنخفضة بالبمموعات المعالجه بالجرعات العالية وبحموعة المراقبة. بينما عند دراسة تأثير الترامادول على مستوى البرولاكتين بالدم كان هناك زيادة ملحوظه بين بحموعات الذكور المعالجه بالترامادول ولكن لم يكن هناك تغير بين بحموعات الإناث. وعند فحص أنسجة المبايض والخصى بالميكروسكوب الضوئي وفحص الغدد النخاميه بالميكروسكوب الألكتروين كانت هناك تغيرات تعكس التغيرات الهرمونية. استنتاج: الأستخدام المزمن للترامادول في الفئران حتى في الجرعات العلاجيه يؤثر بالسلب على الوظيفة التناسلية في كل من الذكور والإناث وكذلك على الوزن أيضاً.

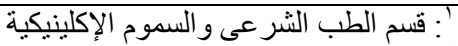

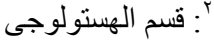
كليه الطب - جامعة عين شمس - القاهرة - مصر 